SLAC-TN-10-051

LCLS-TN-07-03

\title{
Undulator Long Coil Measurement System Tests
}

\author{
Zachary Wolf, Yurii Levashov \\ SLAC
}

April 2, 2007

\begin{abstract}
The first and second field integrals in the LCLS undulators must be below a specified limit. To accurately measure the field integrals, a long coil system is used. This note describes a set of tests which were used to check the performance of the long coil system.
\end{abstract}

\section{$1 \quad$ Introduction $^{1}$}

A long coil measurement system was built to measure the field integrals of the LCLS undulators. The design of the system was described in a previous note ${ }^{2}$. In order to verify that the system is working properly, a set of tests was performed. This note documents the tests and the results.

The maximum values for the field integrals have been specified as follows ${ }^{3}$.

$$
\begin{aligned}
& I_{1 x, y}<40 \times 10^{-6} \mathrm{Tm} \\
& I_{2 x, y}<50 \times 10^{-6} \mathrm{Tm}^{2}
\end{aligned}
$$

In these expressions, $I_{1}$ refers to the first field integral and $I_{2}$ to the second. $x$ refers to the horizontal direction and $y$ to the vertical direction. The long coil measurements must have the sensitivity and accuracy to measure these small field integrals and insure that the limits are met. The measurements are challenging because the coil is only allowed to move by a millimeter or less in order to minimize averaging over a significant volume of field. The resulting signals are very small. This note documents tests which were performed to verify that the system could reliably determine the field integrals.

\section{Measurement Of Field Integrals}

Two tests were done to check the sensitivity and accuracy of the field integral measurements. In the first test, a fluxgate probe with high sensitivity sampled the horizontal field $B_{x}$ and vertical field $B_{y}$ along a line in the $z$ direction. The samples were numerically integrated to calculate the field integrals. This technique let us determine the small background fields from the Earth and nearby objects. The long coil measurement of the same fields was compared to the fluxgate measurements.

In the second set of tests, the long coil was used to measure the first and second field integrals of a small magnet that had its strength measured by independent means. The long coil measurements were compared to the known field integrals. A brief explanation of the measurements follows.

\footnotetext{
${ }^{1}$ Work supported in part by the DOE Contract DE-AC02-76SF00515. This work was performed in support of the LCLS project at SLAC.

${ }^{2}$ Z. Wolf, "Undulator Field Integral Measurements", LCLS-TN-05-22, August 2005.

${ }^{3}$ H. D. Nuhn et al, "LCLS Undulator Requirements", LCLS Specification \# 1.4-001 rev 2.
} 
The field integrals are defined as follows

$$
\begin{aligned}
I_{1 x} & \equiv \int_{0}^{L} B_{x}(z) d z \\
I_{1 y} & \equiv \int_{0}^{L} B_{y}(z) d z \\
I_{2 x} & \equiv \int_{0}^{L} \int_{0}^{z} B_{x}\left(z^{\prime}\right) d z^{\prime} d z \\
I_{2 y} & \equiv \int_{0}^{L} \int_{0}^{z} B_{y}\left(z^{\prime}\right) d z^{\prime} d z
\end{aligned}
$$

The measurements begin at $z=0$, which is approximately at the cell boundary at the entrance end of the undulator. The measurements end at $z=L$, which is approximately at the cell boundary at the exit end of the undulator.

With these definitions, it is straightforward to calculate the field integrals using samples from the fluxgate probe. The fields $B_{x}(z)$ and $B_{y}(z)$ are measured at a large number of $z$ locations. The samples are numerically integrated to calculate the field integrals.

The field integrals are also measured using a long coil. Consider a wire translated parallel to itself horizontally through a magnetic field as shown in figure 1. If the return path is fixed, the

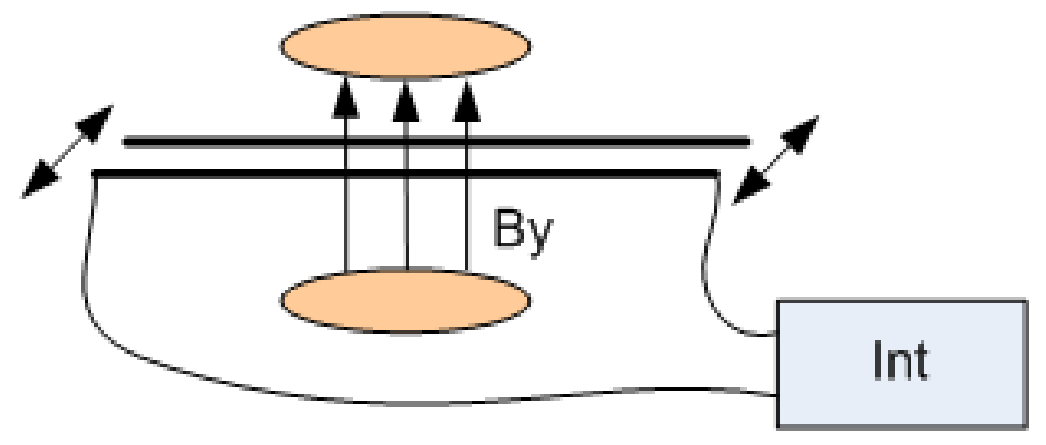

Figure 1: A wire moving through a magnetic field generates a voltage. The voltage is integrated to give the flux change.

magnitude of the voltage induced in the circuit is the time rate of change of the magnetic flux, $V=d \Phi / d t$. The voltage is sent to an integrator whose output is $V T \equiv \int V d t=\int d \Phi=\Delta \Phi$. The integrator output gives the flux change in the circuit. If the wire is moved a distance $\Delta x$, the flux change is given by $\Delta \Phi=\Delta x \int_{0}^{L} B_{y} d z$. The field integral is measured by dividing the integrator output by the distance the wire is moved.

$$
I_{1 y}=\int_{0}^{L} B_{y} d z=\frac{V T}{\Delta x}
$$

For a coil of $N$ turns, the expression becomes

$$
I_{1 y}=\int_{0}^{L} B_{y} d z=\frac{V T}{N \Delta x}
$$

Thus, the first field integral is measured by moving a bundle of wires parallel to itself through the magnetic field. Horizontal motion gives $I_{1 y}$ and vertical motion gives $I_{1 x}$. 
To understand how to use the long coil to calculate second field integrals, we derive a more useful expression by integrating by parts. Temporarily suppressing the $x$ or $y$ subscript, we have

$$
I_{2}=\int_{0}^{L} \int_{0}^{z} B\left(z^{\prime}\right) d z^{\prime} d z
$$

Let $u(z)=\int_{0}^{z} B\left(z^{\prime}\right) d z^{\prime}, d v=d z$. Then $d u=B(z) d z, v=z$. Integrating by parts,

$$
\int_{a}^{b} u d v=\left.u v\right|_{a} ^{b}-\int_{a}^{b} v d u
$$

we find

$$
I_{2}=\left.z \int_{0}^{z} B\left(z^{\prime}\right) d z^{\prime}\right|_{0} ^{L}-\int_{0}^{L} z B(z) d z
$$

Putting the limits into the first term and dropping the primes gives

$$
I_{2}=L \int_{0}^{L} B(z) d z-\int_{0}^{L} z B(z) d z
$$

Adding the field components to the notation, we write the equations for $I_{2 x, y}$ as

$$
\begin{aligned}
& I_{2 x}=\int_{0}^{L}(L-z) B_{x}(z) d z \\
& I_{2 y}=\int_{0}^{L}(L-z) B_{y}(z) d z
\end{aligned}
$$

In this form it is clear that the second field integrals have a weighting factor equal to the distance to the end point of the integration. This linear weighting factor allows easy interpretation for doing measurements with a coil. We hold the exit end of the wire fixed and move the entrance end by a distance $\Delta x$ to measure the second integral of $B_{y}$. This situation is illustrated in figure 2 . In this

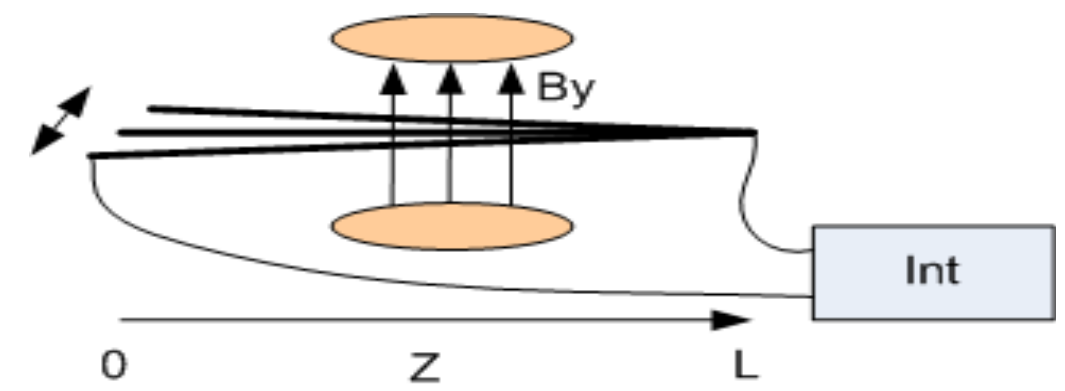

Figure 2: The undulator entrance end of the wire is moved and the exit end is held stationary to measure the second field integral.

case the integrator output, which is equal to the flux change, is given by

$$
V T=\Delta \Phi=\int_{0}^{L} \Delta x \frac{L-z}{L} B_{y}(z) d z
$$

Thus, the second field integral of $B_{y}$ is given by

$$
I_{2 y}=\int_{0}^{L}(L-z) B_{y}(z) d z=\frac{V T L}{\Delta x}
$$


For an $N$ turn coil, this expression becomes

$$
I_{2 y}=\int_{0}^{L}(L-z) B_{y}(z) d z=\frac{V T L}{N \Delta x}
$$

This equation illustrates that the second field integral can be easily obtained from the integrator output when one end of the wire is moved. Moving the wire horizontally gives $I_{2 y}$. Moving the wire vertically gives $I_{2 x}$.

$$
I_{2 x}=\int_{0}^{L}(L-z) B_{x}(z) d z=\frac{V T L}{N \Delta y}
$$

\section{$3 \quad$ Test Results}

\subsection{Fluxgate Probe Test}

A fluxgate probe was used to measure the Earth's field and background fields at our measurement bench. The fluxgate probe had a sensitivity of 10 Volts per Gauss and an accuracy of $0.5 \%$. With such high sensitivity and accuracy, we have a good measurement of a small field with small field integrals. By comparing the long coil measurements to the field integrals from the fluxgate measurements, we establish that the long coil can measure small field integrals.
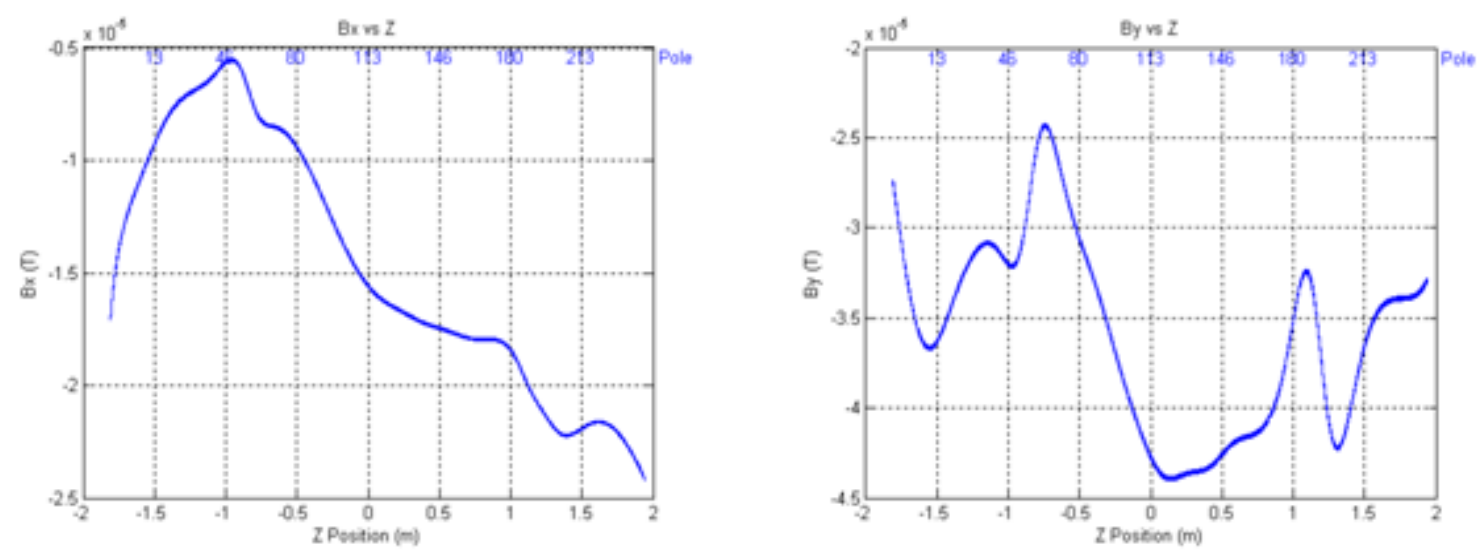

Figure 3: Measured fields along the test bench using a fluxgate probe.

The fields measured by the fluxgate probe are shown in figure 3 . Numerically integrating the fields, we get

$$
\begin{aligned}
& I_{1 x}=-5.50 \times 10^{-5} \mathrm{Tm} \\
& I_{1 y}=-1.35 \times 10^{-4} \mathrm{Tm} \\
& I_{2 x}=-8.28 \times 10^{-5} \mathrm{Tm}^{2} \\
& I_{2 y}=-2.45 \times 10^{-4} \mathrm{Tm}^{2}
\end{aligned}
$$

The long coil was used to measure the field integrals along the same line as the fluxgate probe. 
The long coil gives

$$
\begin{aligned}
& I_{1 x}=-6.24 \times 10^{-5} \mathrm{Tm} \\
& I_{1 y}=-1.47 \times 10^{-4} \mathrm{Tm} \\
& I_{2 x}=-9.88 \times 10^{-5} \mathrm{Tm}^{2} \\
& I_{2 y}=-2.68 \times 10^{-4} \mathrm{Tm}^{2}
\end{aligned}
$$

The long coil field integrals agree with the field integrals from the fluxgate probe at the $10 \%$ level. The exact position of the fluxgate scan is only approximately known to coincide with the long coil position, which we believe is the source of the difference. Our conclusion is that the long coil does have the sensitivity to measure field integrals at the level of the specification limit.

\subsection{Reference Magnet Test}

Another set of tests was performed using magnets of known strength and checking that the long coil system gave the correct answer. Two short magnets were used, one giving a field $B_{x}$ in the horizontal direction, and the other giving a field $B_{y}$ in the vertical direction. The integrated strengths of the magnets were

$$
\begin{aligned}
& B L_{x}=1.65 \times 10^{-3} \mathrm{Tm} \\
& B L_{y}=8.50 \times 10^{-3} \mathrm{Tm}
\end{aligned}
$$

for the horizontal and vertical fields, respectively. The strength of the $B_{y}$ magnet was determined by measuring the field in the gap with a Hall probe and multiplying by the length of the steel plus a gap length. Typically, this method is accurate to around $5 \%$. The $B_{x}$ magnet was very short and had a large gap making the previous technique unusable. Its strength was measured in the Magnetic Measurement Laboratory at SLAC using an independent stretched wire system.

Consider placing the $B_{x}$ and $B_{y}$ magnets at different positions $z_{m}$ along the coil. The position $z_{m}=0$ is at the upstream end of the coil and $z_{m}=L$ is at the downstream end of the coil. This is illustrated in figure 4. For a magnet placed at position $z_{m}$, the field distribution along the $z$ axis is

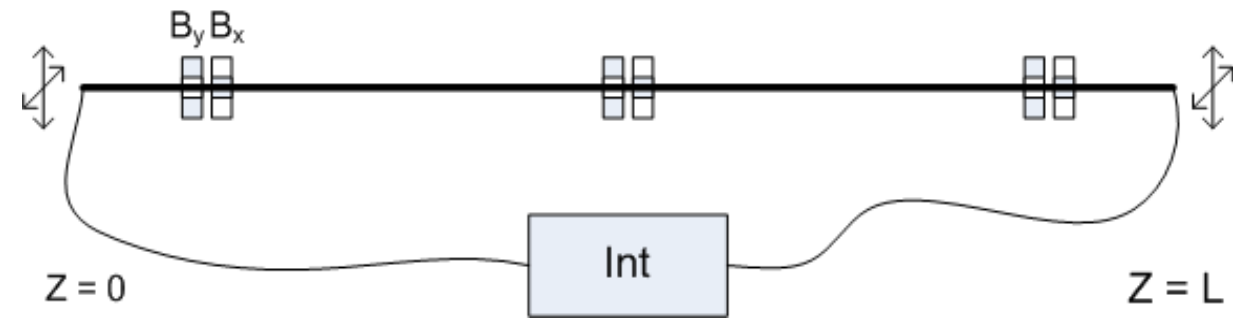

Figure 4: Short magnets of known strength were placed along the coil to check the first and second field integral measurements.

$$
B(z)=B L \delta\left(z-z_{m}\right)
$$

We treat the field distribution of these short magnets as a $\delta$ function. The first integral is given by

$$
I_{1}(z)=B L u\left(z-z_{m}\right)
$$

where $u$ is the unit step function. The second integral is

$$
\begin{aligned}
I_{2}(z) & =0 & & \left(z<z_{m}\right) \\
& =B L\left(z-z_{m}\right) & & \left(z>z_{m}\right)
\end{aligned}
$$


Setting $z=L$, the length of the coil,

$$
\begin{aligned}
& I_{1}=B L \\
& I_{2}=B L\left(L-z_{m}\right)
\end{aligned}
$$

These equations hold for both the $B_{x}$ and $B_{y}$ magnets.

For this test, the $B_{y}$ magnet was placed at $z_{m}=0.10,1.10,2.10$, and 3.30 meters. The coil length is $3.61 \mathrm{~m}$. Inserting these values into the above equations, we get

\begin{tabular}{|c|c|c|c|c|}
\hline & $z_{m}=0.10 \mathrm{~m}$ & $z_{m}=1.10 \mathrm{~m}$ & $z_{m}=2.10 \mathrm{~m}$ & $z_{m}=3.30 \mathrm{~m}$ \\
\hline$I_{1 y}(\mathrm{Tm})$ & $8.50 \times 10^{-3}$ & $8.50 \times 10^{-3}$ & $8.50 \times 10^{-3}$ & $8.50 \times 10^{-3}$ \\
\hline$I_{2 y}\left(\mathrm{Tm}^{2}\right)$ & $2.98 \times 10^{-2}$ & $2.13 \times 10^{-2}$ & $1.28 \times 10^{-2}$ & $0.26 \times 10^{-2}$ \\
\hline
\end{tabular}

The first and second field integrals of $B_{y}$ were measured for each magnet position using the long coil. The measured results are as follows. Note that different current directions were used for some of the measurements. Also included in the tables are results corrected by subtracting the background field integrals given in equations 23 to 26 with no magnet present.

\begin{tabular}{|c|c|c|c|c|}
\hline & $z_{m}=0.10 \mathrm{~m}$ & $z_{m}=1.10 \mathrm{~m}$ & $z_{m}=2.10 \mathrm{~m}$ & $z_{m}=3.30 \mathrm{~m}$ \\
\hline$I_{1 y}(\mathrm{Tm})$ & $8.86 \times 10^{-3}$ & $-9.18 \times 10^{-3}$ & $8.84 \times 10^{-3}$ & $8.79 \times 10^{-3}$ \\
\hline$I_{2 y}\left(\mathrm{Tm}^{2}\right)$ & $3.01 \times 10^{-2}$ & $-2.25 \times 10^{-2}$ & $1.34 \times 10^{-2}$ & $0.35 \times 10^{-2}$ \\
\hline$I_{1 y \text { corr }}(\mathrm{Tm})$ & $9.01 \times 10^{-3}$ & $-9.03 \times 10^{-3}$ & $8.99 \times 10^{-3}$ & $8.94 \times 10^{-3}$ \\
\hline$I_{2 y \text { corr }}\left(\mathrm{Tm}^{2}\right)$ & $3.04 \times 10^{-2}$ & $-2.22 \times 10^{-2}$ & $1.37 \times 10^{-2}$ & $0.38 \times 10^{-2}$ \\
\hline
\end{tabular}

The $B_{x}$ magnet was placed at $z_{m}=0.24,1.24,2.24$, and 3.44 meters. The calculated and measured field integrals are shown below.

\begin{tabular}{|c|c|c|c|c|}
\hline & $z_{m}=0.24 \mathrm{~m}$ & $z_{m}=1.24 \mathrm{~m}$ & $z_{m}=2.24 \mathrm{~m}$ & $z_{m}=3.44 \mathrm{~m}$ \\
\hline$I_{1 x}(\mathrm{Tm})$ & $1.65 \times 10^{-3}$ & $1.65 \times 10^{-3}$ & $1.65 \times 10^{-3}$ & $1.65 \times 10^{-3}$ \\
\hline$I_{2 x}\left(\mathrm{Tm}^{2}\right)$ & $5.56 \times 10^{-3}$ & $3.91 \times 10^{-3}$ & $2.26 \times 10^{-3}$ & $0.28 \times 10^{-3}$ \\
\hline
\end{tabular}

\begin{tabular}{|c|c|c|c|c|}
\hline & $z_{m}=0.24 \mathrm{~m}$ & $z_{m}=1.24 \mathrm{~m}$ & $z_{m}=2.24 \mathrm{~m}$ & $z_{m}=3.44 \mathrm{~m}$ \\
\hline$I_{1 x}(\mathrm{Tm})$ & $-1.72 \times 10^{-3}$ & $-1.77 \times 10^{-3}$ & $1.63 \times 10^{-3}$ & $1.61 \times 10^{-3}$ \\
\hline$I_{2 x}\left(\mathrm{Tm}^{2}\right)$ & $-5.50 \times 10^{-3}$ & $-3.97 \times 10^{-3}$ & $2.26 \times 10^{-3}$ & $0.49 \times 10^{-3}$ \\
\hline$I_{1 x \text { corr }}(\mathrm{Tm})$ & $-1.66 \times 10^{-3}$ & $-1.71 \times 10^{-3}$ & $1.69 \times 10^{-3}$ & $1.67 \times 10^{-3}$ \\
\hline$I_{2 x \text { corr }}\left(\mathrm{Tm}^{2}\right)$ & $-5.40 \times 10^{-3}$ & $-3.87 \times 10^{-3}$ & $2.36 \times 10^{-3}$ & $0.59 \times 10^{-3}$ \\
\hline
\end{tabular}

These measurements confirm that the long coil system is measuring the first and second field integrals at the accuracy level of roughly $6 \%$, which is primarily dominated by the method used to determine the strength of the $B_{y}$ magnet. We conclude that the system is functioning properly. It is capable of confirming that the field integrals are below the specified limits.

\section{Conclusion}

A long coil system was constructed to measure the first and second field integrals of the LCLS undulators. The long coil measurements of the background fields were compared to field integrals obtained by sampling the background fields and numerically calculating the integrals. This test showed that the long coil has the sensitivity required to measure at the levels specified for the field integrals.

Tests were also performed by making long coil measurements of short magnets of known strength placed at various positions The long coil measurements agreed with the known field integrals obtained by independent measurements and calculation. Our tests showed that the long coil measurements are a valid way to determine whether the LCLS undulator field integrals are below the specified limits. 